\title{
Single-arm confirmatory trial of laparoscopy-assisted total or proximal gastrectomy with nodal dissection for clinical stage I gastric cancer: Japan Clinical Oncology Group study JCOG1401
}

\author{
Hitoshi Katai ${ }^{1}$ - Junki Mizusawa ${ }^{2} \cdot$ Hiroshi Katayama $^{2} \cdot$ Chikara Kunisaki $^{3}$. Shinichi Sakuramoto ${ }^{4}$. Noriyuki Inaki ${ }^{5}$. \\ Takahiro Kinoshita $^{6} \cdot$ Yoshiaki Iwasaki $^{7} \cdot$ Kazunari Misawa $^{8} \cdot$ Nobuhiro Takiguchi $^{9} \cdot$ Masahide Kaji $^{10}$. \\ Hiroshi Okitsu ${ }^{11}$. Takaki Yoshikawa ${ }^{1}$. Masanori Terashima ${ }^{12}$. On behalf of the Stomach Cancer Study Group of \\ Japan Clinical Oncology Group
}

Received: 16 October 2018 / Accepted: 18 January 2019 / Published online: 20 February 2019

(c) The International Gastric Cancer Association and The Japanese Gastric Cancer Association 2019

\begin{abstract}
Backgrounds Laparoscopy-assisted distal gastrectomy (LADG) for gastric cancer is safe and feasible. In contrast, no prospective study evaluating the safety and efficacy of laparoscopy-assisted total gastrectomy (LATG) or laparoscopy-assisted proximal gastrectomy (LAPG) has been completed. We conducted a single-arm confirmatory trial to evaluate the safety of LATG/LAPG for clinical stage I (T1N0/T1N1/T2N0) proximal gastric cancer.

Methods The extent of lymphadenectomy was selected based on the Japanese Gastric Cancer Treatment Guidelines. The mini-laparotomy incision was required to be $\leq 6 \mathrm{~cm}$. The primary endpoint was the proportion of grade $2-4$ (CTCAE ver. 4.0) esophagojejunal anastomotic leakage. The planned sample size was 245 considering a threshold of $8 \%$ and one-sided alpha of $2.5 \%$.

Results Between April 2015 and February 2017, 244 eligible patients were enrolled. LATG/LAPG was performed in 195/49. The proportion of conversions was $1.7 \%$. Clinical T1N0/T1N1/T2N0 was 212/9/23. The extents of lymphadenectomy were as follows: D1+: 229; D2: 15. The median operation time was $309 \mathrm{~min}$ (IQR 265-353). The median blood loss was $30 \mathrm{ml}$ (IQR 10-86). Grade 2-4 esophagojejunal anastomotic leakage was 2.5\% (6/244; 95\% CI 0.9-5.3). The overall proportion of in-hospital grade 3-4 adverse events was $29 \%$ (71/244). The proportions of intraabdominal abscess and pancreatic fistula were $3.7 \%$ and $2.0 \%$, respectively. There were no treatment-related deaths.

Conclusions This trial confirmed the safety of LATG/LAPG. After the non-inferiority of LADG is confirmed in our phase III trial (JCOG0912), LATG/LAPG is expected to be established as one of the standard treatments for clinical stage I gastric cancer.
\end{abstract}

Keywords Gastric cancer · Laparoscopic surgery · Gastrectomy · Clinical trial

\section{Introduction}

Laparoscopy-assisted distal gastrectomy (LADG) for gastric cancer is established as being safe and feasible. Moreover, several phase III studies have attempted to confirm non-inferiority of LADG to open surgery for early disease. Our group [the Stomach Cancer Study Group of the Japan Clinical Oncology Group (JCOG)] has been engaged in establishing

Hitoshi Katai

hkatai@ncc.go.jp

Extended author information available on the last page of the article evidence for laparoscopic gastrectomy. We conducted a multicenter phase II trial (JCOG0703) and confirmed the safety of LADG for early gastric cancer [1]. Then, we proceeded to a randomized phase III trial to confirm the noninferiority of LADG to open distal gastrectomy (ODG) in terms of relapse-free survival (JCOG0912) [2, 3]. Patient accrual has already been completed, and the primary analysis results will be published in 2019. Laparoscopy-assisted total gastrectomy (LATG) or laparoscopy-assisted proximal gastrectomy (LAPG) was not included in the treatment protocol of JCOG0703 or JCOG0912, as the surgical techniques for LATG and LAPG had not yet been standardized and, 
therefore, these techniques were not widely used compared to LADG.

Recently, a standard procedure for LATG and LAPG has been established, and the proportions of LATG and LAPG performed among laparoscopy-assisted gastrectomies have increased. Although some retrospective studies have demonstrated the safety of LATG compared to open total gastrectomy (OTG), we are unable to arrive at any definitive conclusions regarding the safety or survival benefit of LATG because of the small sample sizes in these studies. So far, no prospective study has been completed to determine either the safety or long-term survival benefit of LATG. Therefore, we were prompted to conduct a clinical trial to evaluate the safety and efficacy of LATG and LAPG for early gastric cancer.

Considering that the results of the phase III trial being conducted to evaluate the long-term outcome of LADG (JCOG0912) would also validate the usefulness of LATG/ LAPG, we conducted this single-arm confirmatory trial to evaluate the safety of LATG/LAPG for patients with clinical stage I (T1N0/T1N1/T2N0) proximal gastric cancer.

\section{Methods}

\section{Study design}

This multi-institutional single-arm confirmatory trial is being conducted within the framework of the JCOG Stomach Cancer Study Group. The trial was designed to demonstrate the safety of LATG and LAPG with nodal dissection for patients with clinical stage I gastric cancer, in terms of the proportion of patients with esophagojejunal anastomotic leakage. Only surgeons specialized in both procedures at 35 Japanese institutions participated in the study. The study protocol was approved by the Protocol Review Committee of the JCOG and also approved and overseen by the institutional review board of each participating hospital. The design of the trial was reported previously [4].

\section{Participants}

Patients with histologically proven gastric adenocarcinoma, clinical T1N0, T1N $(+)$, or T2N0 according to the 14th edition of the Japanese Classification of Gastric Carcinoma (3rd English edition), who were treatable by total gastrectomy or proximal gastrectomy [5], and in whom endoscopic mucosal resection (EMR) or endoscopic submucosal dissection (ESD) was not indicated, were initially eligible for this study.

The $\mathrm{T}$ category was evaluated by upper gastrointestinal endoscopy. Multi-detector row CT was used for diagnosis of the $\mathrm{T}$ category, $\mathrm{N}$ category, and $\mathrm{M}$ category.
The detailed eligibility criteria for the study are shown in Table 1. All patients gave written informed consent.

\section{Procedure}

LATG or LAPG was performed in all the patients. The extent of nodal dissection was determined according to the surgical $\mathrm{T}$ and $\mathrm{N}$ stages, which were determined based on the 4th version of the Japanese Gastric Cancer Treatment Guidelines [6]. D1 or more extensive dissection was applied for clinical stage IA tumor, and D2 dissection for clinical stage IB tumor. Splenectomy and bursectomy were not allowed, but preservation of the omentum and/or celiac branch of the vagus nerve was discretionary. For patients requiring total gastrectomy, only Roux-en-Y reconstruction was allowed. For patients requiring proximal gastrectomy, only double-tract or jejunal-interposition reconstruction was allowed. Mini-laparotomy was limited to 1 site, and the length of the skin incision was to be less than or equal to $6 \mathrm{~cm}$. When the skin incision required extension to more than $6 \mathrm{~cm}$, the case was considered conversion to open surgery. If the intraoperative findings revealed a tumor stage II or greater, the LATG or LAPG was converted to open surgery. The operative time, blood loss, and all post-operative morbidities during hospitalization were included on the case report forms for prospective data collection. Postoperative morbidity was described according to the Common Terminology Criteria for Adverse Events (CTCAE) ver. 4.0 [7], and the JCOG postoperative complication criteria based on the Clavien-Dindo classification [8]. The type of post-operative analgesia, such as epidural anesthesia, was not specified. Usage of analgesics on post-operative days 5-10 was recorded.

\section{Quality control of surgery}

All of the following criteria (1)-(3) for a certified surgeon in laparoscopic gastrectomy must be fulfilled.

1. Experience of performing 30 or more laparoscopic gastrectomies.

2. Certification in the area of gastric cancer by either a surgical quality assurance (QA) committee or the Japan Society for Endoscopic Surgery.

3. Either (i) or (ii) must be fulfilled.

i. Experience in performing 15 or more esophagojejunal anastomoses using a linear stapler.

ii. Experience in performing 15 or more esophagojejunal anastomoses using a circular stapler. 
Table 1 Eligibility criteria

Inclusion criteria

(1) Histologically proven gastric papillary adenocarcinoma (pap), tubular adenocarcinoma (tub1, tub2), poorly differentiated adenocarcinoma (por1, por2), signet-ring cell carcinoma (sig), or mucinous adenocarcinoma (muc)

(2) Clinical T1N0, T1N(+)*, or T2N0 according to the 14th edition of the Japanese Classification of Gastric Carcinoma (3rd English edition)

(3) In cases of $\mathrm{T} 2 \mathrm{~N} 0$, tumor not involving the greater curvature

(4) In cases without prior history of endoscopic mucosal resection (EMR) or endoscopic submucosal dissection (ESD), either cN(+) or cN0 with no indication for EMR or ESD

(5) In cases with prior history of EMR or ESD, all of the following conditions fulfilled

(i) Pathological findings indicative of the need for additional gastrectomy

(ii) Within 91 days of EMR or ESD

(iii) No perforation resulting from EMR or ESD

(6) R0 resection expected by total gastrectomy or proximal gastrectomy

(7) No invasion of the duodenum or esophagus

(8) Age 20-80 years

(9) Performance Status (Eastern Cooperative Oncology Group) 0 or 1

(10) Body Mass Index less than 30

(11) No history of upper abdominal surgery or intestinal resection, except for appendectomy

(12) No history of prior chemotherapy or radiation therapy for any other malignancies. Cases with a history of hormone therapy, but at least 1 year after the last administration, are eligible

(13) Sufficient organ functions

(i) White blood cell count $\geq 3000 / \mathrm{mm}^{3}$

(ii) Platelet count $\geq 100,000 / \mathrm{mm}^{3}$

(iii) Serum total bilirubin level $\leq 2.0 \mathrm{mg} / \mathrm{dl}$

(iv) Serum aspartate transaminase level $\leq 100 \mathrm{IU} / 1$

(v) Serum alanine transaminase level $\leq 100 \mathrm{IU} / \mathrm{l}$

(vi) Serum creatinine level $\leq 1.5 \mathrm{mg} / \mathrm{dl}$

(14) Written informed consent available

Exclusion criteria

(1) Synchronous or metachronous (within 5 years) malignancies, except for carcinoma in situ or mucosal tumors curatively treated by local therapy

(2) Infectious disease necessitating systemic therapy

(3) Body temperature $\geq 38^{\circ} \mathrm{C}$

(4) Pregnancy, possible pregnancy, or within 28 days after delivery or breastfeeding

(5) Severe psychiatric disease

(6) Continuous systemic steroid or immunosuppressive drug therapy

(7) Unstable angina pectoris, or history of myocardial infarction within the previous 6 months

(8) Poorly controlled hypertension

(9) Poorly controlled diabetes mellitus despite continuous insulin use

(10) Severe respiratory disease requiring continuous oxygen therapy

Gastric cancer is staged according to the Japanese Classification of Gastric Carcinoma-14th Japanese version, which corresponds to the 3rd English edition

*Corresponding to T1N1 according to the 13th edition of the Japanese Classification of Gastric Carcinoma (2nd English edition)

A surgical QA committee, which was credentialed by the study chair, judged the video recording of the laparoscopic gastrectomy. Certified surgeons performed the LATG or LAPG as an operator or teaching assistant.

\section{Intraoperative photographs and video recording}

A surgical QA committee performed a central peer review of the surgical procedure by photographing all the patients. A 
central peer review was performed every 6-12 months. Intraoperative video recordings of arbitrarily selected patients were also played in a group conference that was held 3 times a year, to share the operation procedure.

\section{Follow-up}

All registered patients were followed up for at least 5 years. Adjuvant chemotherapy with S-1 for 1 year was recommended for patients who were finally diagnosed as having pathological stage II, IIIA, or IIIB disease. Measurements of tumor markers, chest radiography, upper gastrointestinal endoscopy, and enhanced abdominal computed tomography were performed at least every year and for at least 5 years.

\section{Outcomes}

The primary endpoint was the proportion of patients with esophagojejunal anastomotic leakage. This was defined as the proportion of patients with grade 2 (CTCAE $v$ 4.0) [7] or greater esophagojejunal anastomotic leakage among all operated patients. A grade 2 or greater esophagojejunal anastomotic leakage is defined in CTCAE v 4.0 as "symptomatic; medical intervention indicated," and corresponds to a grade II or greater gastrointestinal anastomotic leak described in the JCOG Postoperative Complication Criteria according to the Clavien-Dindo Classification [8]. Grade II gastrointestinal anastomotic leakage was defined as "medical management (e.g., antibiotics) or enteral/intravenous nutrition (including total parenteral nutrition) indicated." Anastomotic leakage caused by other post-operative complications (e.g., pancreatic fistula) was also included as an event.

The secondary endpoints were overall survival, relapsefree survival, proportion of completed LATGs or LAPGs, proportion of conversions to open surgery, adverse events, and short-term clinical outcomes. The proportion of completed LATGs or LAPGs was defined as the proportion of patients in whom LATG or LAPG was completed without conversion to open surgery among all the surgically treated patients. The proportion of conversions to open surgery was defined as the proportion of conversions among patients diagnosed as having clinical stage IA or IB disease prior to gastrectomy. The short-term clinical outcomes included: (1) the time from the end of surgery until the first passage of flatus, (2) proportion of patients requesting analgesia on post-operative days $5-10,(3)$ the highest body temperature during the first 3 days after surgery, and (4) the highest body temperature during the hospitalization.

\section{Study design and statistical analysis}

This was a trial performed to demonstrate the safety of LATG/LAPG in terms of the proportion of patients developing esophagojejunal anastomotic leakage among all patients undergoing gastrectomy. When the proportion of patients developing this post-operative complication was low, as expected, we considered that LATG or LAPG was as safe as open total or proximal gastrectomy. Once the safety of LATG and LAPG is demonstrated, and LADG becomes a treatment option based on the results of JCOG0912, LATG and LAPG would become established as being among the standard treatment options for clinical stage IA/IB gastric cancer. According to previous large-scale studies, the proportion of patients undergoing open total or proximal gastrectomy who develop esophagojejunal anastomotic leakage is in the range of $2-3 \%[9,10]$.

Similarly, according to retrospective data, the reported proportion of patients undergoing LATG or LAPG developing esophagojejunal anastomotic leakage is in the range of $0-4 \%$ [11-14]. Taking these reports into account, the sample size was calculated as 242 patients to obtain $90 \%$ power, with the hypothesis that the primary endpoint would have an expected value of $3 \%$ and threshold value of $8 \%$ in onesided testing at a $2.5 \%$ significance level. The total sample size was set at 245 patients by assuming that a few patients will not undergo gastrectomy. The confidence interval for proportion was estimated by the Clopper-Pearson method. All statistical analyses were conducted at the JCOG Data Center using SAS 9.4 (SAS Institute, Cary, NC, USA). The data presented in this article are as of May 2017.

No interim analysis was planned. When treatment-related death or severe (grade 4) surgical morbidity was observed in 8 or more patients, the registration was to be suspended and the JCOG Data and Safety Monitoring Committee was given the responsibility to determine whether the trial could restart.

In-house interim monitoring was performed every 6 months by the JCOG Data Center, to evaluate and improve the study progress, data integrity, and patient safety. This study is registered in the UMIN Clinical Trials Registry as UMIN000017155 (http://www.umin.ac.jp/ctr/).

\section{Results}

\section{Patient characteristics}

Between April 2015 and February 2017, 246 patients were enrolled from 35 institutions in Japan (Fig. 1). One patient was excluded from this analysis because the patient was misregistered before the study was approved by the institutional review board. The other patient was deemed as ineligible and excluded from the safety analysis because he developed perforation as a complication of ESD.

The patient demographics are summarized in Table 2. The median age was 67 years. The male-to-female ratio was 
Fig. 1 Trial profile

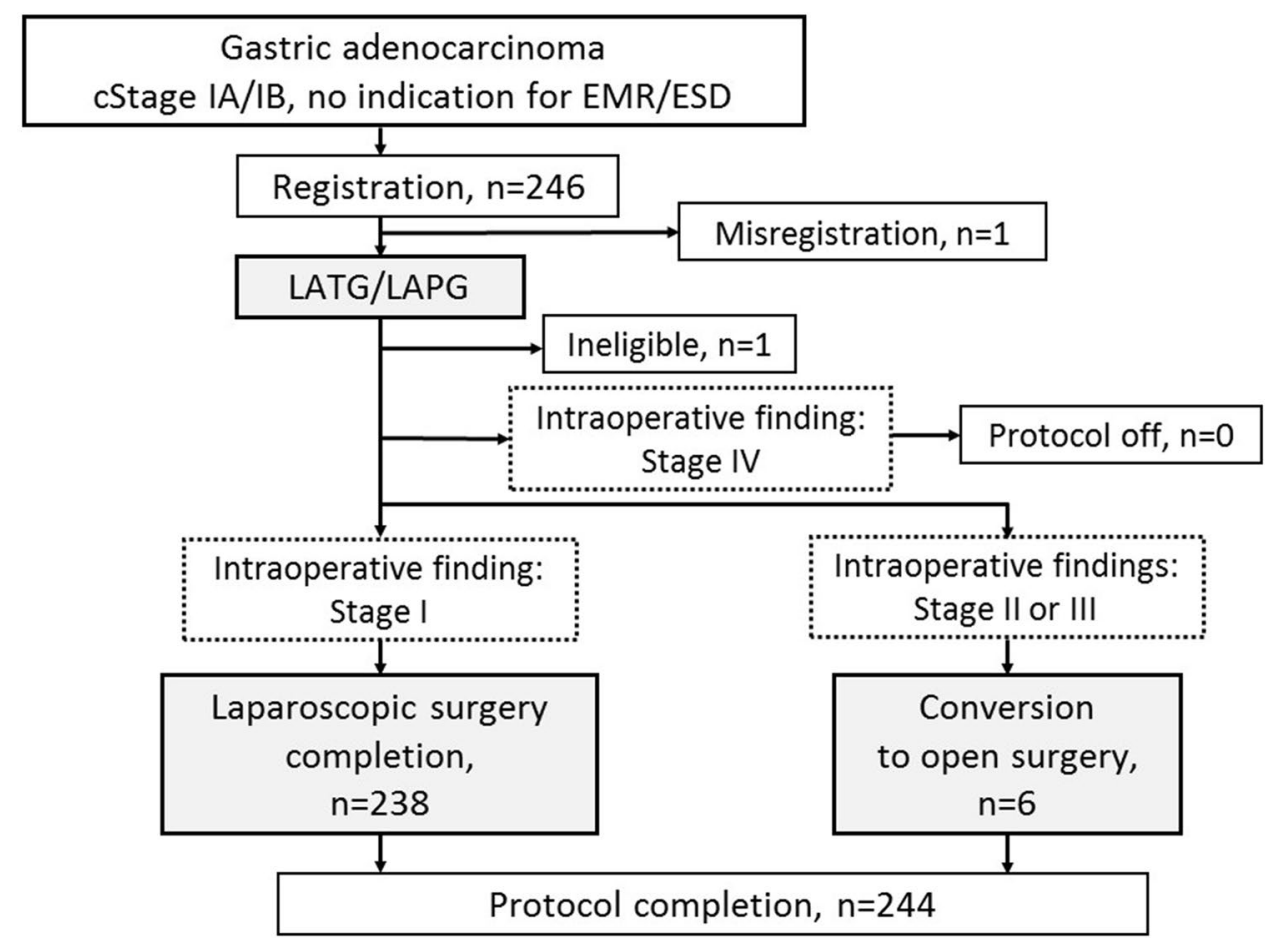

Table 2 Baseline characteristics (all enrolled patients)

\begin{tabular}{ll}
\hline $\begin{array}{l}\text { Age, median } \\
\left(\mathrm{IQR}^{\mathrm{a}}\right)\end{array}$ & 67 \\
(Range) & $(60-72)$ \\
Gender & $(31-80)$ \\
Male & $173(70.6 \%)$ \\
Female & $72(29.4 \%)$ \\
ECOG performance status & \\
0 & $244(99.6 \%)$ \\
1 & $1(0.4 \%)$ \\
BMI (kg/m $\left.{ }^{2}\right)$, median & 22.8 \\
$($ IQR) & $(21.1-24.7)$ \\
(Range) & $(16.1-29.2)$ \\
Clinical T and N stages** & \\
T1N0 & $212(86.5 \%)$ \\
T1N(+) & $9(3.7 \%)$ \\
T2(MP)N0 & $24(9.8 \%)$ \\
Clinical stage** & \\
IA & $213(86.9 \%)$ \\
IB & $32(13.1 \%)$ \\
Prior ESD & \\
Yes & $39(15.9 \%)$ \\
No & $206(84.1 \%)$ \\
\hline
\end{tabular}

${ }^{\mathrm{a}}$ Interquartile range

**Japanese Classification of Gastric Carcinoma, 14th Japanese version
$2.4: 1$, and $86.5 \%$ of the patients had cStage IA (cT1N0) disease. The median body mass index was 22.8 .

\section{Operative procedures}

All the operations were performed with curative intent. The surgical procedures and outcomes are summarized in Table 3.

The median operative time was $309 \mathrm{~min}$. The median blood loss was $30 \mathrm{ml}$, and blood transfusions were required in 5 patients $(2.0 \%)$.

LATG was performed in 195 (79.9\%) patients and LAPG in 49 (20.1\%) patients. D1+ was performed in 229 (93.9\%) patients and D2 in 15 (6.1\%) patients. Roux-en-Y was performed in 195 (79.9\%) patients and was the predominant type of reconstruction performed, followed by double-tract in $45(18.4 \%)$, and interposition in $4(1.6 \%)$ patients.

The median length of the skin incision was $4.2 \mathrm{~cm}$. The laparoscopic procedure was successfully completed in 238 [97.5\%; 95\% confidence interval (CI), 94.7-99.1] of the 244 patients, which was significantly better than the expected value (70\%).

The surgical procedure was converted from a laparoscopic procedure to an open procedure in 4 patients with a preoperative diagnosis of clinical $\mathrm{T} 1 \mathrm{~N} 0, \mathrm{~T} 1 \mathrm{~N}(+)$, and $\mathrm{T} 2 \mathrm{~N} 0$; consequently, the proportion of conversions was $1.7 \%$ (95\% CI, 0.5-4.2), which was lower than expected (5\%). The reasons for conversion were bleeding ( 2 patients), intraoperative diagnosis of stage II or III tumor (2 patients). 
Table 3 Operative details (all eligible patients)

\begin{tabular}{ll}
\hline Operative time (min), median & 309 \\
(IQR) & $(265-353)$ \\
(Range) & $(181-574)$ \\
Blood loss (ml), median & 30 \\
(IQR) & $(10-86)$ \\
(Range) & $(0-3428)$ \\
Operative procedures & \\
LATG & $195(79.9 \%)$ \\
LAPG & $49(20.1 \%)$ \\
Extent of lymph node dissection & \\
D0 & $0(0 \%)$ \\
D1 & $0(0 \%)$ \\
D1+ & $229(93.9 \%)$ \\
D2 & $15(6.1 \%)$ \\
D2+ & $0(0 \%)$ \\
Number of harvested lymph nodes, median (IQR) & $50(35-64)$ \\
Reconstruction method & \\
Roux-en-Y & $195(79.9 \%)$ \\
Double tract & $45(18.4 \%)$ \\
Interposition & $4(1.6 \%)$ \\
Length of skin incision (cm), Median (IQR) & $4.2(4.0-5.0)$ \\
Completion of laparoscopic procedure $(n=244)$ & $238(97.5 \%)$ \\
95\% CI & $94.7-99.1 \%$ \\
Conversion to open procedure $(n=242)$ & $4(1.7 \%)$ \\
95\% CI & $0.5-4.2 \%$ \\
\hline
\end{tabular}

Completion of LADG was counted for all operated patients

Conversion to open procedure was counted for all patients diagnosed before gastrectomy as having clinical T1N0, T1N(+), T2N0 disease

\section{Short-term clinical outcomes (Table 4)}

Flatus passage was recognized in all the patients while they were in hospital. The median time from the end of surgery until the first passage of flatus was 3 days. Analgesia was required on post-operative days 5-10 in 118 of the 244 patients $(48.4 \%)$, even though the expected value was $20 \%$ or lower.

The highest body temperature during the first 3 days after surgery was recorded; the median was $37.7{ }^{\circ} \mathrm{C}$ (range, 36.4-39.4) on day $1,37.4^{\circ} \mathrm{C}$ (range, 36.3-39.2) on post-operative day 2 , and $37.1{ }^{\circ} \mathrm{C}$ (range, 35.9-38.9) on post-operative day 3 . The median highest body temperature during the entire period of hospitalization was $37.8{ }^{\circ} \mathrm{C}$ (range, $36.8-40.0$ ); the expected value was less than $38.0^{\circ} \mathrm{C}$.

\section{Histological findings}

The histological findings are shown in Table 5. Early gastric cancer (T1) was confirmed by histopathology in about $80 \%$ of the patients. The overall proportion of patients with nodal involvement was $16.4 \%$ (40 out of 244 patients).

\section{Operative complications and deaths (Table 6)}

One patient developed a grade 3 or 4 intraoperative adverse event (splenic vein injury). The proportion of patients with esophagojejunal anastomotic leakage (grade 2 or more), which was the primary endpoint, was $2.5 \%$ (6/244), with a 95\% CI of $0.9-5.3 \%$, which was significantly lower than prespecified threshold of $8 \%$ (one-sided $p=0.0002$ ).

The overall proportion of in-hospital adverse events (grade 3 or 4 according to CTCAEv4.0) was $29.1 \%$ (71/244). Grade 3 or higher pancreatic fistula, post-operative bleeding, abscess, and esophagojejunal anastomotic leakage were observed in 5 patients, 1 patient, 9 patients, and 4 patients, respectively. The proportion of patients with grade 3 or 4 serum AST/ALT elevation was high (20.9\%). None of the patients required re-operation. The post-operative mortality was zero.
Table 4 Short-term clinical outcomes (all eligible patients)

\begin{tabular}{ll}
\hline & Median (IQR) \\
\hline $\begin{array}{l}\text { Days from the end of surgery until the first passage of flatus } \\
\text { Analgesic use on POD 5-10 }\end{array}$ & 3.0 days (3.0-4.0 days) \\
Highest body temperature during the first 3 days & $118(48.4 \%)$ \\
Body temperature during the first 3 days & $37.8^{\circ} \mathrm{C}(37.5-38.2)$ \\
POD 1 & \\
POD2 & $37.7^{\circ} \mathrm{C}(37.3-38.1)$ \\
POD3 & $37.4{ }^{\circ} \mathrm{C}(37.1-37.8)$ \\
Re-operation & $37.1{ }^{\circ} \mathrm{C}(36.8-37.4)$ \\
Red blood cell transfusions during hospital stay & $0(0 \%)$ \\
Operation-related death & $5(2.0 \%)$ \\
\hline
\end{tabular}

${ }^{\text {aPost-operative day }}$ 
Table 5 Histological findings (all eligible patients)

\begin{tabular}{|c|c|}
\hline & $(n=244)$ \\
\hline Tumor size $(\mathrm{cm})$, median & 3.0 \\
\hline (IQR) & $(2.2-5.0)$ \\
\hline (Range) & $(0.6-18.0)$ \\
\hline \multicolumn{2}{|l|}{ Histological type } \\
\hline Pap & $5(2.0 \%)$ \\
\hline Tub1 & $65(26.6 \%)$ \\
\hline Tub2 & $69(28.3 \%)$ \\
\hline Por & $74(30.3 \%)$ \\
\hline Sig & $24(9.8 \%)$ \\
\hline Muc & $0(0 \%)$ \\
\hline Others & $7(2.9 \%)$ \\
\hline \multicolumn{2}{|l|}{ T stage } \\
\hline T1a & $71(29.1 \%)$ \\
\hline $\mathrm{T} 1 \mathrm{~b}$ & $119(48.8 \%)$ \\
\hline $\mathrm{T} 2$ & $31(12.7 \%)$ \\
\hline $\mathrm{T} 3$ & $15(6.1 \%)$ \\
\hline $\mathrm{T} 4 \mathrm{a}$ & $8(3.3 \%)$ \\
\hline $\mathrm{T} 4 \mathrm{~b}$ & $0(0 \%)$ \\
\hline \multicolumn{2}{|l|}{ N stage (13th) } \\
\hline N0 & $204(83.6 \%)$ \\
\hline N1 & $30(12.3 \%)$ \\
\hline $\mathrm{N} 2$ & $10(4.1 \%)$ \\
\hline N3 & $0(0 \%)$ \\
\hline \multicolumn{2}{|l|}{ N stage (14th) } \\
\hline No & $204(83.6 \%)$ \\
\hline N1 & $25(10.2 \%)$ \\
\hline $\mathrm{N} 2$ & $12(4.9 \%)$ \\
\hline N3a & $2(0.8 \%)$ \\
\hline $\mathrm{N} 3 \mathrm{~b}$ & $1(0.4 \%)$ \\
\hline pH1 & $0(0 \%)$ \\
\hline $\mathrm{pP} 1$ & $0(0 \%)$ \\
\hline \multicolumn{2}{|l|}{ Lavage cytology } \\
\hline CY0 & $70(28.7 \%)$ \\
\hline CY1 & $0(0 \%)$ \\
\hline CYX & $174(71.3 \%)$ \\
\hline \multicolumn{2}{|l|}{ Proximal margin } \\
\hline PM0 & $244(100 \%)$ \\
\hline PM1 & $0(0 \%)$ \\
\hline \multicolumn{2}{|l|}{ Distal margin } \\
\hline DM0 & $244(100 \%)$ \\
\hline DM1 & $0(0 \%)$ \\
\hline \multicolumn{2}{|l|}{$\mathrm{R}$ classification } \\
\hline R0 & $244(100 \%)$ \\
\hline $\mathrm{R} 1$ & $0(0 \%)$ \\
\hline $\mathrm{R} 2$ & $0(0 \%)$ \\
\hline \multicolumn{2}{|l|}{ Stage (13th) } \\
\hline IA & $170(69.7 \%)$ \\
\hline IB & $47(19.3 \%)$ \\
\hline II & $17(7.0 \%)$ \\
\hline IIIA & $8(3.3 \%)$ \\
\hline
\end{tabular}

Table 5 (continued)

\begin{tabular}{ll}
\hline & $(n=244)$ \\
\hline IIIB & $2(0.8 \%)$ \\
IV & $0(0 \%)$ \\
Stage (14th) & \\
IA & $170(69.7 \%)$ \\
IB & $36(14.8 \%)$ \\
IIA & $20(8.2 \%)$ \\
IIB & $10(4.1 \%)$ \\
IIIA & $5(2.0 \%)$ \\
IIIB & $1(0.4 \%)$ \\
IIIC & $2(0.8 \%)$ \\
IV & $0(0 \%)$ \\
Number of harvested lymph nodes, median & 41.5 \\
(IQR) & $(30-61)$ \\
(Range) & $(7-122)$ \\
Number of metastatic lymph nodes, median & 0 \\
(IQR) & $(0-0)$ \\
(Range) & $(0-21)$ \\
\hline
\end{tabular}

\section{Discussion}

The present study was a single-arm trial performed to determine the safety of LATG/LAPG. The proportion of patients developing esophagojejunal anastomotic leakage was low in this study, clearly satisfying our hypothesis in regard to the safety of LATG/LAPG. Moreover, proportion of patients with other major complications was also low, the post-operative recovery was favorable, the conversion rate was low, and the post-operative mortality was zero. There was no difference between LATG and LAPG in terms of the incidence of esophagojejunal leakage. These results indicate the safety as well as feasibility of LATG/LAPG.

One possible reason for the success of this trial is that the surgeons performing the LATG/LAPG procedures were limited to those who had the experience of performing at least 30 laparoscopic gastrectomy procedures, including 15 or more esophagojejunal anastomoses. Moreover, the technical skill level of the attending surgeons was guaranteed and confirmed by all the group members by checking the video recordings and photographs. These selection criteria might be too strict, but were acceptable by our previous reports $[1,3]$. Conversely, some studies have demonstrated a high complication rate associated with LATG when this procedure was not performed by credentialed surgeons [14-17]. Thus, while LATG/LAPG is safe and feasible, it must be performed by surgeons who are sufficiently trained and skilled in the procedure.

Other possible reasons might be selection biases. At first, the eligibility criteria of BMI and the extent of nodal dissection, namely $\mathrm{D} 2$ or $\mathrm{D} 1+$, which was related to the technical 
Table 6 Grade $2-4$ adverse events

\begin{tabular}{|c|c|c|c|c|c|c|}
\hline \multirow[t]{2}{*}{ CTCAE v4.0 } & \multicolumn{2}{|l|}{$\begin{array}{l}\text { LAPG } \\
N=49\end{array}$} & \multicolumn{2}{|l|}{$\begin{array}{l}\text { LATG } \\
N=195\end{array}$} & \multicolumn{2}{|l|}{$\begin{array}{l}\text { Total } \\
N=244\end{array}$} \\
\hline & G2-4 & G3-4 & G2-4 & G3-4 & G2-4 & G3-4 \\
\hline Intraoperative events & 0 & 0 & $1(0.5 \%)$ & $1(0.5 \%)$ & $1(0.4 \%)$ & $1(0.4 \%)$ \\
\hline In-hospital events & $29(59.2 \%)$ & $11(22.4 \%)$ & $133(68.2 \%)$ & $60(30.8 \%)$ & $162(66.4 \%)$ & $71(29.1 \%)$ \\
\hline \multicolumn{7}{|l|}{ Non-hematological surgical } \\
\hline Pancreatic fistula & 0 & 0 & $9(4.6 \%)$ & $5(2.6 \%)$ & $9(3.7 \%)$ & $5(2.0 \%)$ \\
\hline Bleeding & 0 & 0 & $4(2.1 \%)$ & $1(0.5 \%)$ & $4(1.6 \%)$ & $1(0.4 \%)$ \\
\hline Abscess & 0 & 0 & $9(4.6 \%)$ & $9(4.6 \%)$ & $9(3.7 \%)$ & $9(3.7 \%)$ \\
\hline Esophageal anastomotic leak & $2(4.1 \%)$ & $1(2.0 \%)$ & $4(2.1 \%)$ & $3(1.5 \%)$ & $6(2.5 \%)$ & $4(1.6 \%)$ \\
\hline Gastrointestinal anastomotic leak & 0 & 0 & $1(0.5 \%)$ & $1(0.5 \%)$ & $1(0.4 \%)$ & $1(0.4 \%)$ \\
\hline Small intestinal anastomotic leak & 0 & 0 & $2(1.0 \%)$ & $2(1.0 \%)$ & $2(0.8 \%)$ & $2(0.8 \%)$ \\
\hline Stenosis & 0 & 0 & 0 & 0 & 0 & 0 \\
\hline Delayed gastric emptying & 0 & 0 & 0 & 0 & 0 & 0 \\
\hline Gastro-esophageal regurgitation & 0 & 0 & $1(0.5 \%)$ & 0 & $1(0.4 \%)$ & 0 \\
\hline Obstruction & $1(2.0 \%)$ & 0 & $3(1.5 \%)$ & $3(1.5 \%)$ & $4(1.6 \%)$ & $3(1.2 \%)$ \\
\hline Ileus & 0 & 0 & $3(1.5 \%)$ & 0 & $3(1.2 \%)$ & 0 \\
\hline Thromboembolic event & 0 & 0 & $1(0.5 \%)$ & $1(0.5 \%)$ & $1(0.4 \%)$ & $1(0.4 \%)$ \\
\hline Pneumonia & 0 & 0 & $6(3.1 \%)$ & $3(1.5 \%)$ & $6(2.5 \%)$ & $3(1.2 \%)$ \\
\hline Chyle leakage & 0 & 0 & $1(0.5 \%)$ & 0 & $1(0.4 \%)$ & 0 \\
\hline Wound infection & 0 & 0 & $5(2.6 \%)$ & $1(0.5 \%)$ & $5(2.0 \%)$ & $1(0.4 \%)$ \\
\hline Wound dehiscence & 0 & 0 & $2(1.0 \%)$ & 0 & $2(0.8 \%)$ & 0 \\
\hline \multicolumn{7}{|l|}{ Non-hematological non-surgical } \\
\hline Hypoalbuminemia $^{\mathrm{a}}$ & $20(40.8 \%)$ & $0(0 \%)$ & $93(49.2 \%)$ & $3(1.6 \%)$ & $113(47.5 \%)$ & $3(1.3 \%)$ \\
\hline Hyperbilirubinemia & $2(4.1 \%)$ & $1(2.0 \%)$ & $8(4.1 \%)$ & $1(0.5 \%)$ & $10(4.1 \%)$ & $2(0.8 \%)$ \\
\hline AST/ALT increased & $14(28.6 \%)$ & $7(14.3 \%)$ & $66(33.8 \%)$ & $44(22.6 \%)$ & $80(32.8 \%)$ & $51(20.9 \%)$ \\
\hline Creatinine increased & 0 & 0 & $2(1.0 \%)$ & 0 & $2(0.8 \%)$ & 0 \\
\hline Hypernatremia & 0 & 0 & 0 & 0 & 0 & 0 \\
\hline Hyponatremia & $3(6.1 \%)$ & $3(6.1 \%)$ & $3(1.5 \%)$ & $3(1.5 \%)$ & $6(2.5 \%)$ & $6(2.5 \%)$ \\
\hline Hyperkalemia & $2(4.1 \%)$ & $1(2.0 \%)$ & $6(3.1 \%)$ & 0 & $8(3.3 \%)$ & $1(0 \cdot 4 \%)$ \\
\hline \multicolumn{7}{|l|}{ Hematological } \\
\hline Leukopenia & $1(2.0 \%)$ & 0 & $1(0.5 \%)$ & 0 & $2(0.8 \%)$ & 0 \\
\hline Anemia & $3(6.1 \%)$ & $0(0 \%)$ & $29(14.9 \%)$ & $6(3.1 \%)$ & $32(13.1 \%)$ & $6(2.5 \%)$ \\
\hline Thrombocytopenia & $1(2.0 \%)$ & 0 & $1(0.5 \%)$ & 0 & $2(0.8 \%)$ & 0 \\
\hline
\end{tabular}

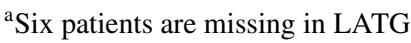

difficulty of the surgery, were discussed. The BMI of the present cohort was 22.8, which was identical to that in our phase III study, JCOG0912. The proportion of D2 lymphadenectomy in this study was also identical to that in JCOG0912. These results suggest that obese patients or T2N0 patients who needed D2 were not intentionally excluded from the study. Second, age and gender were also taken into consideration. Elderly patients have more co-morbidities, which can easily lead to surgical complications. Male gender is a risk factor for the development of complications after surgery. In the present study, the median patient age was 67 years and the male-to-female ratio was $2.4: 1$, suggesting that LATG/ LAPG was more likely to be performed in older and male patients, who may have more adverse events, compared to the JCOG0912 study. However, these proportions were identical to those determined from a retrospective analysis of more than 100,000 patients from the nationwide registry of the Japanese Gastric Cancer Association (2001-2007) [18]. The IQR of the BMI in this study population was identical to that of OTG in previous publications from a nationwide web-based database [16, 17]. The baseline characteristics of this study population would be closer to the real-world cohort for gastric cancer in Japan than those of the study population of JCOG0912. Thus, the excellent results of this study are unlikely to be influenced by any selection biases.

The quality of the surgical procedures in the present study is also important. The proportion of patients with any type of leakage (grade 3-4) was 3\% in this study, which 
was lower than that reported in previous publications from the nationwide web-based database $(5.3-5.4 \%)$ [16, 17]. In addition, the proportion of patients developing anastomotic leakage was considered to be equivalent to or lower than that of the proportion developing this complication after ODG (3.2-6.1\%) [16, 17]. The proportions of patients developing other complications such as intraabdominal abscess (3.7\%) and pancreatic fistula (2.0\%), which were mainly related to the lymph node dissection, were lower than those associated with OTG and LTG as determined from the nationwide web-based database $(6.4-6.9 \%)$ [16, 17]. The operative times were longer and the blood loss was lower in patients undergoing LATG/LAPG than in those undergoing OTG, as reported in previous investigations of data from the nationwide web-based database $[16,17]$. The operative time was shorter and the blood loss was smaller in this study than in the aforementioned study based on the nationwide webbased database $[16,17]$. Thus, the frequency of complications, the operative time, and the volume of blood loss observed in this study were acceptable compared to previous reports.

In regard to post-operative recovery, LATG/LAPG was associated with a 1-day delay in the passage of the first flatus than LADG, which was also the case for ODG determined in JCOG0912. The use of analgesics after five post-operative days was more than we expected, and the same as that in the cases undergoing LADG reported from JCOG0912. The median highest body temperature was less than $38.0{ }^{\circ} \mathrm{C}$. These results demonstrate a more favorable post-operative recovery after laparoscopic surgery.

The target population of this trial, JCOG1401, was patients with stage I gastric cancer and was the same as that for the JCOG0912 study. The surgical procedure performed (distal gastrectomy, total gastrectomy or proximal gastrectomy) was selected based on the tumor location and not by the stage of the tumor. The technical difficulty level is known to influence the treatment efficacy. Although the reconstruction method in LATG/LAPG is more difficult than that in LADG, the technical difficulty level of lymph node dissection in early gastric cancer does not differ between LATG/LAPG and LADG. The presumed 5-year overall survival was $90 \%$ in the study population of JCOG0912, and the 5 -year overall survival in the target population of JCOG1401 was identical to that of JCOG0912 [19, 20].

We assumed that evidence for the efficacy of LADG could be extrapolated to LATG/LAPG if the safety of LATG/LAPG could be confirmed and LADG becomes a valid treatment option based on the results of JCOG0912.

In conclusion, this trial confirmed the safety and feasibility of LATG/LAPG in terms of the incidence of esophagojejunal anastomotic leakage, when the procedure is conducted by credentialed surgeons. Once the non-inferiority of LADG is confirmed in our phase III trial (ODG versus
LADG: JCOG0912), LATG and LAPG could come to be considered as being standard treatments for clinical stage I gastric cancer in Japan.

Funding The study was supported in part by the National Cancer Center Research and Development Funds (26-A-4, 29-A-3), Grant-inAid for Clinical Cancer Research H26-053, and AMED under Grant numbers JP18ck0106317, JP15ck0106056.

\section{Compliance with ethical standards}

Conflict of interest We certify that we have no conflicts of interests to declare.

Human rights statement All procedures followed were in accordance with the ethical standards of the responsible committee on human experimentation (institutional and national) and with the Helsinki Declaration of 1964 and later versions.

Informed consent Informed consent was obtained from all patients included in the study.

\section{References}

1. Katai H, Sasako M, Fukuda H, Nakamura K, Hiki N, Saka M, et al. Safety and feasibility of laparoscopy-assisted distal gastrectomy with suprapancreatic nodal dissection for clinical stage I gastric cancer: a multicenter phase II trial (JCOG 0703). Gastric Cancer. 2010;13(4):238-44.

2. Nakamura K, Katai H, Mizusawa J, Yoshikawa T, Ando M, Terashima M, et al. A phase III study of laparoscopy-assisted versus open distal gastrectomy with nodal dissection for clinical stage IA/IB gastric cancer (JCOG0912). Jpn J Clin Oncol. 2013;43(3):324-7.

3. Katai H, Mizusawa J, Katayama H, Takagi M, Yoshikawa T, Fukagawa T, et al. Short-term surgical outcomes from a phase III study of laparoscopy-assisted versus open distal gastrectomy with nodal dissection for clinical stage IA/IB gastric cancer: Japan Clinical Oncology Group Study JCOG0912. Gastric Cancer. 2017;20(4):699-708.

4. Kataoka K, Katai H, Mizusawa J, Katayama H, Nakamura K, Morita S, et al. Non-randomized confirmatory trial of laparoscopy-assisted total gastrectomy and proximal gastrectomy with nodal dissection for clinical stage I gastric cancer: Japan Clinical Oncology Group study JCOG1401. J Gastric Cancer. 2016;16(2):93-7.

5. Japanese Gastric Cancer A. Japanese classification of gastric carcinoma: 3rd English edition. Gastric Cancer. 2011;14(2):101-12.

6. Japanese Gastric Cancer A. Japanese gastric cancer treatment guidelines 2014 (ver. 4). Gastric Cancer. 2017;20(1):1-19.

7. Institute NC. Common terminology criteria for adverse events (CTCAE) version 4.0. Bethesda: National Cancer Institute; 2015.

8. Katayama H, Kurokawa Y, Nakamura K, Ito H, Kanemitsu Y, Masuda N, et al. Extended Clavien-Dindo classification of surgical complications: Japan Clinical Oncology Group postoperative complications criteria. Surg Today. 2016;46(6):668-85.

9. Deguchi Y, Fukagawa T, Morita S, Ohashi M, Saka M, Katai H. Identification of risk factors for esophagojejunal anastomotic leakage after gastric surgery. World J Surg. 2012;36(7):1617-22. 
10. Sano T, Sasako M, Mizusawa J, Yamamoto S, Katai H, Yoshikawa $\mathrm{T}$, et al. Randomized controlled trial to evaluate splenectomy in total gastrectomy for proximal gastric carcinoma. Ann Surg. 2017;265(2):277-83.

11. Ebihara Y, Okushiba S, Kawarada Y, Kitashiro S, Katoh H. Outcome of functional end-to-end esophagojejunostomy in totally laparoscopic total gastrectomy. Langenbeck's Arch Surg. 2013;398(3):475-9.

12. Haverkamp L, Weijs TJ, van der Sluis PC, van der Tweel I, Ruurda JP, van Hillegersberg R. Laparoscopic total gastrectomy versus open total gastrectomy for cancer: a systematic review and metaanalysis. Surg Endosc. 2013;27(5):1509-20.

13. Lee MS, Lee JH, Park DJ, Lee HJ, Kim HH, Yang HK. Comparison of short- and long-term outcomes of laparoscopic-assisted total gastrectomy and open total gastrectomy in gastric cancer patients. Surg Endosc. 2013;27(7):2598-605.

14. Wada N, Kurokawa Y, Takiguchi S, Takahashi T, Yamasaki M, Miyata $\mathrm{H}$, et al. Feasibility of laparoscopy-assisted total gastrectomy in patients with clinical stage I gastric cancer. Gastric Cancer. 2014;17(1):137-40.

15. Oshi M, Kunisaki C, Miyamoto H, Kosaka T, Akiyama H, Endo I. Risk factors for anastomotic leakage of esophagojejunostomy after laparoscopy-assisted total gastrectomy for gastric cancer. Dig Surg. 2018;35(1):28-34.

16. Etoh T, Honda M, Kumamaru H, Miyata H, Yoshida K, Kodera Y, et al. Morbidity and mortality from a propensity score-matched, prospective cohort study of laparoscopic versus open total gastrectomy for gastric cancer: data from a nationwide web-based database. Surg Endosc. 2018;32(6):2766-73.

17. Kodera Y, Yoshida K, Kumamaru H, Kakeji Y, Hiki N, Etoh $\mathrm{T}$, et al. Introducing laparoscopic total gastrectomy for gastric cancer in general practice: a retrospective cohort study based on a nationwide registry database in Japan. Gastric Cancer. 2019;22(1):202-13.

18. Katai H, Ishikawa T, Akazawa K, Isobe Y, Miyashiro I, Oda I, et al. Five-year survival analysis of surgically resected gastric cancer cases in Japan: a retrospective analysis of more than 100,000 patients from the nationwide registry of the Japanese Gastric Cancer Association (2001-2007). Gastric Cancer. 2018;21(1):144-54.

19. Kunisaki C, Akiyama H, Nomura M, Matsuda G, Otsuka Y, Ono $\mathrm{H}$, et al. Surgical outcomes for early gastric cancer in the upper third of the stomach. J Am Coll Surg. 2005;200(1):15-9.

20. Katai H, Morita S, Saka M, Taniguchi H, Fukagawa T. Long-term outcome after proximal gastrectomy with jejunal interposition for suspected early cancer in the upper third of the stomach. Br J Surg. 2010;97(4):558-62.

Publisher's Note Springer Nature remains neutral with regard to jurisdictional claims in published maps and institutional affiliations.

\section{Affiliations}

\section{Hitoshi Katai ${ }^{1}$ (1) - Junki Mizusawa ${ }^{2} \cdot$ Hiroshi Katayama $^{2} \cdot$ Chikara Kunisaki $^{3} \cdot$ Shinichi Sakuramoto $^{4} \cdot$ Noriyuki Inaki $^{5}$. Takahiro Kinoshita $^{6} \cdot$ Yoshiaki Iwasaki $^{7} \cdot K$ azunari Misawa ${ }^{8} \cdot$ Nobuhiro Takiguchi $^{9} \cdot$ Masahide Kaji $^{10}$. Hiroshi Okitsu ${ }^{11}$. Takaki Yoshikawa ${ }^{1}$. Masanori Terashima ${ }^{12}$. On behalf of the Stomach Cancer Study Group of Japan Clinical Oncology Group}

1 Department of Gastric Surgery, National Cancer Center Hospital, 5-1-1 Tsukiji, chuo-ku, Tokyo 104-0045, Japan

2 Japan Clinical Oncology Group Data Center/Operations Office, National Cancer Center Hospital, Tokyo, Japan

3 Department of Surgery, Yokohama City University, Yokohama, Japan

4 Department of Gastroenterological Surgery, Saitama Medical University International Medical Center, Hidaka, Japan

5 Department of Gastroenterological Surgery, Ishikawa Prefectural Central Hospital, Kanazawa, Japan

6 Department of Gastric Surgery, National Cancer Center Hospital East, Kashiwa, Japan
7 Department of Surgery, IMS Tokyo-Katsushika General Hospital, Tokyo, Japan

8 Department of Gastroenterological Surgery, Aichi Cancer Center Hospital, Nagoya, Japan

9 Department of Gastroenterological Surgery, Chiba Cancer Center, Chiba, Japan

10 Department of Surgery, Toyama Prefectural Central Hospital, Toyama, Japan

11 Department of Surgery, Tokushima Red Cross Hospital, Komatsushima, Japan

12 Division of Gastric Surgery, Shizuoka Cancer Center, Nagaizumi, Japan 